\title{
SCHUR MULTIPLIERS AND OPERATOR-VALUED FOGUEL-HANKEL OPERATORS
}

\author{
C. BADEA AND V.I. PAULSEN
}

\begin{abstract}
We show that some matrices are Schur multipliers and this is applied to obtain classes of operator-valued Foguel-Hankel operators similar to contractions. This provides partial answers to a problem of K. Davidson and the second author concerning CAR-valued Foguel-Hankel operators.
\end{abstract}

\section{Introduction}

An example of a polynomially bounded operator on Hilbert space not similar to a contraction was found recently by Pisier [Pi]. An operatortheoretic proof that certain CAR-valued Foguel-Hankel operators are polynomially bounded operators but not similar to contractions was given by Davidson and Paulsen [DP]. It is still an open question [DP] to characterize operators in this family which are similar to contractions.

The aim of this note is to prove some partial results concerning this open problem. The present note is a sequel of $[\mathrm{DP}]$ where this problem is studied. A certain familiarity with [DP] is supposed. For the convenience of the reader some notation and known facts are recalled below.

1.1. Background. We denote by $H$ a separable Hilbert space and by $B(H)$ the $\mathrm{C}^{*}$-algebra of all bounded and linear operators on $H$. An operator $T \in B(H)$ is said to be power bounded if

$$
\sup _{n \in \mathbb{Z}_{+}}\left\|T^{n}\right\|<+\infty
$$

1991 Mathematics Subject Classification. 47A20, 47A56, 47B35.

Key words and phrases. Similarity to contraction, Foguel-Hankel operators, Schur multipliers. 
and polynomially bounded if there exists a constant $K$ such that

$$
\|p(T)\| \leq K\|p\|_{\infty}
$$

for each analytic polynomial $p$. Here

$$
\|p\|_{\infty}:=\sup \{|p(z)|:|z| \leq 1\} .
$$

We say that $T$ is similar to a contraction if there is an invertible operator $L \in B(H)$ such that

$$
\left\|L^{-1} T L\right\| \leq 1 .
$$

The following implications hold :

$$
\begin{aligned}
T \text { similar to a contraction } & \Rightarrow T \text { polynomially bounded } \\
& \Rightarrow T \text { power bounded } .
\end{aligned}
$$

The first implication follows from von Neumann's $[\mathrm{vN}]$ inequality

$$
\|p(C)\| \leq\|p\|_{\infty},
$$

valid for each contraction $C \in B(H)$. The second implication is clear from (1.1) and (1.2).

It was proved by Paulsen [Pa1] that $T \in B(H)$ is similar to a contraction if and only if $T$ is completely polynomially bounded, that is, there exists a constant $K$ such that

$$
\left\|\left[p_{i j}(T)\right]_{1 \leq i, j \leq n}\right\| \leq K \sup \left\{\left\|\left[p_{i j}(z)\right]_{1 \leq i, j \leq n}\right\|:|z| \leq 1\right\},
$$

for all positive integers $n$ and all $n \times n$ matrices $\left[p_{i j}\right]_{1 \leq i, j \leq n}$ with polynomial entries. Recall that $\left[p_{i j}(T)\right]_{1 \leq i, j \leq n}$ is identified with an operator acting on the direct sum of $n$ copies of the corresponding Hilbert space in a natural way.

No implication in (1.3) can be reversed. The first power bounded operator not similar to a contraction was constructed by Foguel $[\mathrm{F}]$. His counterexample has the form

$$
R(X)=R\left(S^{*}, S ; X\right)=\left[\begin{array}{cc}
S^{*} & X \\
0 & S
\end{array}\right],
$$


where $S$ denotes the unilateral shift on $\ell_{2}$ and $X$ was a suitable diagonal projection onto a subspace of $\ell_{2}$. We will call Foguel operators the operators of type (1.4).

Lebow [L] proved that the example constructed by Foguel is not polynomially bounded. Other examples of power bounded, not polynomially bounded operators are in [Da, Pe2, Bo].

A Foguel-Hankel operator is a Foguel operator (1.4) with

$$
X=\left[a_{i+j}\right]_{i, j \geq 0}=\Gamma_{f}
$$

a Hankel operator with symbol $f$ (cf. [DP]). The study of FoguelHankel operators was initiated by Foias and Williams [FW] and Peller [Pe1]. It follows from the work of Peller [Pe1], Bourgain [B] and Aleksandrov and Peller [AP] that these Foguel-Hankel operators are similar to contractions whenever they are polynomially bounded. Both conditions are equivalent to $f^{\prime} \in \mathrm{BMOA}$, that is with the boundedness of

$$
\Gamma_{f^{\prime}}=\left[(i+j+1) a_{i+j}\right]_{i, j \geq 0} .
$$

However, it is still unknown if a general Foguel operator is similar to a contraction whenever it is polynomially bounded. See [Fe], where this is related to computing a certain Ext group.

The first example of a polynomially bounded operator not similar to a contraction was found by Pisier [Pi]. His example is a CAR-valued Foguel-Hankel operator which we introduce below.

1.2. CAR-valued Foguel-Hankel operators. Let $\Lambda$ be a function from $H$ into $B(H)$ satisfying the CAR - canonical anticommutation relations : for all $u, v \in H$,

$$
\Lambda(u) \Lambda(v)+\Lambda(v) \Lambda(u)=0
$$

and

$$
\Lambda(u) \Lambda(v)^{*}+\Lambda(v)^{*} \Lambda(u)=(u, v) I .
$$

The range of $\Lambda$ is isometric to Hilbert space. Let $\left\{e_{n}\right\}_{n \geq 0}$ be an orthonormal basis for $H$, and let $C_{n}=\Lambda\left(e_{n}\right)$ for $n \geq 0$. For an arbitrary 
sequence $\alpha=\left(\alpha_{0}, \alpha_{1}, \ldots\right)$ in $\ell^{2}$, let

$$
Y_{\alpha}=\left[\alpha_{i+j} C_{i+j}\right]
$$

be a CAR-valued Hankel operator. Let

$$
R\left(Y_{\alpha}\right)=R\left(S^{*(\infty)}, S^{(\infty)} ; Y_{\alpha}\right)=\left[\begin{array}{cc}
S^{*(\infty)} & Y_{\alpha} \\
0 & S^{(\infty)}
\end{array}\right]
$$

be the corresponding $C A R$-valued Foguel-Hankel operator [Pi], [DP].

The initial choice of $\alpha$ made by Pisier was $\alpha_{2^{k-1}}=1$ for $k \geq 0$ and $\alpha_{i}=0$ otherwise. In this case $R\left(Y_{\alpha}\right)$ is polynomially bounded but not completely polynomially bounded.

The following more general result holds (cf. [Pi], [DP]).

For a fixed sequence $\alpha=\left(\alpha_{0}, \alpha_{1}, \ldots\right) \in \ell_{2}$, let

$$
A=A(\alpha):=\sup _{k \geq 0}(k+1)^{2} \sum_{i \geq k}\left|\alpha_{i}\right|^{2}
$$

and

$$
B_{2}=B_{2}(\alpha):=\sum_{k \geq 0}(k+1)^{2}\left|\alpha_{k}\right|^{2} .
$$

The operator $R\left(Y_{\alpha}\right)$ is polynomially bounded if and only if $A$ is finite. If $R\left(Y_{\alpha}\right)$ is similar to a contraction, then $B_{2}$ is finite.

It is an open problem $[\mathrm{DP}]$ to characterize in terms of the sequence $\alpha$ when $R\left(Y_{\alpha}\right)$ is similar to a contraction. In particular, it is not known if $B_{2}(\alpha)$ finite implies the similarity of $R\left(Y_{\alpha}\right)$ to a contraction. Note [DP, p. 163] that

$$
B_{2}(\alpha)=\left\|\Gamma_{F^{\prime}}\right\|^{2}=\left\|(i+j+1) \alpha_{i+j} C_{i+j}\right\|^{2},
$$

where the operator-valued symbol $F$ given by

$$
F(z)=\sum_{n \geq 0} \alpha_{n} C_{n} z^{-n-1}
$$

is such that $\Gamma_{F}=Y_{\alpha}$.

We refer to $[\mathrm{Pi}],[\mathrm{DP}],[\mathrm{D}]$ for more information and for the undefined terms. 
1.3. Organization of the paper. The main results are stated in the next section. Section three contains some useful results about Schur multipliers. In the fourth section, a sufficient condition for similarity is given. These results are used to prove the main results in the last section.

\section{MAin RESUlts}

We use notations as above. The first two results give sufficient conditions for similarity to a contraction of an operator-valued Foguel operator. Although these results are implicit in the work of $[\mathrm{FW}]$ and $[\mathrm{DP}]$, they do not seem to have been stated elsewhere.

Theorem 2.1. Let $X \in B\left(\ell_{2}(H)\right)$ with matrix $X=\left[X_{i j}\right]_{i, j \geq 0}, X_{i j} \in$ $B(H)$, with respect to a fixed orthonormal basis. For each $n \geq 1$ set

$$
A^{(n)}(X)_{i j}=X_{i j}+X_{i-1, j+1}+\ldots X_{i-\min (i, n-1), j+\min (i, n-1)}
$$

and let $A^{(n)}(X)$ be the matrix $\left[A_{i j}^{(n)}(X)_{i j}\right]_{i, j \geq 0}$. If

$$
\sup _{n \geq 1}\left\|A^{(n)}(X)\right\|<+\infty
$$

then the operator-valued Foguel operator

$$
R\left(S^{*(\infty)}, S^{(\infty)} ; X\right)=\left[\begin{array}{cc}
S^{*(\infty)} & X \\
0 & S^{(\infty)}
\end{array}\right] \in B\left(\ell_{2}(H) \oplus \ell_{2}(H)\right)
$$

is similar to a contraction.

In the case of operator-valued Foguel-Hankel operators the following holds.

Theorem 2.2. Let $\Gamma=\left[\Gamma_{i+j}\right]_{i, j \geq 0}$ be an operator-valued Hankel operator, $\Gamma_{k} \in B(H)$. The operator-valued Foguel-Hankel operator

$$
R\left(S^{*(\infty)}, S^{(\infty)} ; \Gamma\right)=\left[\begin{array}{cc}
S^{*(\infty)} & \Gamma \\
0 & S^{(\infty)}
\end{array}\right] \in B\left(\ell_{2}(H) \oplus \ell_{2}(H)\right)
$$

is similar to a contraction if any of the following operators

$$
\begin{gathered}
\Gamma D-D^{*} \Gamma=\left[(j-i) \Gamma_{i+j-1}\right]_{i, j \geq 0}, \\
\Gamma D=\left[j \Gamma_{i+j-1}\right]_{i, j \geq 0},
\end{gathered}
$$


or

$$
D^{*} \Gamma=\left[i \Gamma_{i+j-1}\right]_{i, j \geq 0}
$$

is a bounded operator. Here $\Gamma_{-1}=0$ and $D=\left[(i+1) \delta_{i+1}^{j}\right]_{i, j \geq 0}$ is the differentiation operator.

If $\Gamma=\Gamma_{f}=\left(a_{i+j}\right)$ is a scalar Hankel operator with symbol $f$, then boundedness of any one of the three operators above is equivalent to boundedness of the other two and this occurs if and only if $f^{\prime} \in$ BMOA. This is a consequence of the fact that both conditions are equivalent to similarity to a contraction of the Foguel-Hankel operator. This, as was remarked in $[\mathrm{AP}]$, also follows from [JP].

For operator-valued Hankels, the situation is much more complicated as is shown in $[\mathrm{DP}]$. A sufficient condition for similarity to a contraction for an operator-valued Foguel-Hankel operator was given by Blower [Bl] in terms of Carleson measures.

In the case of CAR-valued Foguel-Hankel operators, we still do not know if $B_{2}(\alpha)<+\infty$ implies the similarity to a contraction of $R\left(Y_{\alpha}\right)$. Theorem 2.2 and the Schur multipliers results of the next section will imply the following results.

Theorem 2.3 (log $\log$ condition). Let $\varepsilon>0$. Suppose

$$
\sum_{k \geq 1}(k+1)^{2}[\log (k+1)]^{2}[\log (\log (k+1))]^{2+\varepsilon}\left|\alpha_{k}\right|^{2}<+\infty .
$$

Then the CAR-valued Foguel-Hankel operator $R\left(Y_{\alpha}\right)=R\left(S^{*(\infty)}, S^{(\infty)} ; Y_{\alpha}\right)$ is similar to a contraction.

Since the logarithm goes to infinity less quickly than any power, we obtain the following consequences.

Corollary 2.4 (log condition). Let $\varepsilon>0$. Suppose

$$
\sum_{k \geq 0}(k+1)^{2}[\log (k+1)]^{2+\varepsilon}\left|\alpha_{k}\right|^{2}<+\infty .
$$

Then the CAR-valued Foguel-Hankel operator $R\left(Y_{\alpha}\right)$ is similar to a contraction.

and 
Corollary 2.5 (power condition). Let $\varepsilon>0$. Suppose

$$
B_{2+\varepsilon}(\alpha):=\sum_{k \geq 0}(k+1)^{2+\varepsilon}\left|\alpha_{k}\right|^{2}<+\infty .
$$

Then the CAR-valued Foguel-Hankel operator $R\left(Y_{\alpha}\right)$ is similar to a contraction.

A proof of Corollary 2.5 can be given by combining results from [DP] and $[\mathrm{P}]$. A different proof in the case $\varepsilon=1$ can be found in $[\mathrm{Ba}]$.

\section{SCHUR MULTipliers}

Let $A=\left[a_{i j}\right]_{i, j \geq 1}$ and $B=\left[b_{i j}\right]_{i, j \geq 1}$ be two matrices of the same size (finite or infinite). The Schur product of $A$ and $B$ is defined to be the matrix of elementwise products

$$
A * B=\left[a_{i j} b_{i j}\right]_{i, j \geq 1} .
$$

For $M \in B\left(\ell_{2}\right)$ we let $\mathcal{S}_{M}$ denote the Schur multiplication map by $M$ on $B\left(\ell_{2}\right), \mathcal{S}_{M}(A)=M * A$. Then $M$ is said to be a Schur multiplier if

$$
\left\|\mathcal{S}_{M}: B\left(\ell_{2}\right) \rightarrow B\left(\ell_{2}\right)\right\|<+\infty .
$$

If $M=\left[m_{i j}\right]$ is a Schur multiplier and the iterated row and column limits

$$
\lim _{j \rightarrow \infty}\left(\lim _{i \rightarrow \infty} m_{i j}\right)=C \text { and } \lim _{i \rightarrow \infty}\left(\lim _{j \rightarrow \infty} m_{i j}\right)=R
$$

exist, then $[\mathrm{Be}] C=R$. This shows in particular that

$$
\left[\frac{j-i}{i+j+1}\right]_{i, j \geq 1}
$$

is not a Schur multiplier.

Theorem 3.1. Let $\left(a_{n}\right)_{n \geq 1}$ be a sequence of reals which converges to 0. Set

$$
b_{n}=\Delta^{1}\left(a_{n}\right)=a_{n}-a_{n+1} \text { and } c_{n}=\Delta^{2}\left(a_{n}\right)=a_{n}-2 a_{n+1}+a_{n+2} .
$$

If the sequences $\left(a_{n} / n\right),\left(b_{n}\right)$ and $\left(n c_{n}\right)$ are all absolutely summable, then the matrix

is a Schur multiplier.

$$
\left[\frac{(j-i) a_{i+j}}{i+j+1}\right]_{i, j \geq 1}
$$


Proof. The proof will be based on the following criterion due to Bennett [Be, Theorem 8.6] : if $M=\left[m_{i j}\right]_{i, j \geq 1}$ satisfies

$$
\lim _{i} m_{i j}=\lim _{j} m_{i j}=0
$$

and

$$
\sum_{i, j=1}^{+\infty}\left|m_{i, j}-m_{i, j+1}-m_{i+1, j}+m_{i+1, j+1}\right|<+\infty,
$$

then $M$ is a Schur multiplier.

Let $m_{i j}=(j-i) a_{i+j} /(i+j+1)$. We have

$$
\lim _{i} m_{i j}=\lim _{j} m_{i j}=0 .
$$

In order to prove (3.1), we write

$$
\begin{gathered}
\sum_{i, j=1}^{\infty}\left|m_{i, j}-m_{i, j+1}-m_{i+1, j}+m_{i+1, j+1}\right| \\
=\sum_{n=1}^{\infty} \sum_{i+j=n}\left|\frac{(j-i) a_{i+j}}{i+j+1}-\frac{2(j-i) a_{i+j+1}}{i+j+2}+\frac{(j-i) a_{i+j+2}}{i+j+3}\right| \\
\leq \sum_{n=1}^{\infty} n(n-1)\left|\frac{a_{n}}{n+1}-\frac{2 a_{n+1}}{n+2}+\frac{a_{n+2}}{n+3}\right| \\
=\sum_{n=1}^{\infty} n(n-1)\left|\frac{c_{n}}{n+2}+\frac{1}{n+2}\left[\frac{a_{n}}{n+1}-\frac{a_{n+2}}{n+3}\right]\right| \\
=\sum_{n=1}^{\infty} n(n-1)\left|\frac{c_{n}}{n+2}+\frac{b_{n}+b_{n+1}}{(n+1)(n+2)}+\frac{2 a_{n+2}}{(n+1)(n+2)(n+3)}\right| \\
\leq \sum_{n \geq 1} n\left|c_{n}\right|+\sum_{n \geq 1}\left|b_{n}\right|+\sum_{n \geq 1}\left|b_{n+1}\right|+2 \sum_{n \geq 1} \frac{\left|a_{n+2}\right|}{n+2} .
\end{gathered}
$$

All four sums are convergent by hypothesis.

Corollary 3.2. Let $\varepsilon>0$. The matrices

$$
E_{\varepsilon}=\left[\frac{j-i}{(i+j+1)(\log (i+j+1))^{1+\varepsilon}}\right]_{i, j \geq 1}
$$

and

$$
F_{\varepsilon}=\left[\frac{j-i}{(i+j+1)(\log (i+j+1))(\log \log (i+j+1))^{1+\varepsilon}}\right]_{i, j \geq 1}
$$


are Schur multipliers.

Proof. Theorem 3.1 applies for the sequences

$$
a_{n}=\frac{1}{(\log n)^{1+\varepsilon}}, n \geq 2
$$

and

$$
a_{n}=\frac{1}{(\log n)[\log (\log n)]^{1+\varepsilon}}, n \geq 2 .
$$

We give the proof only for the second sequence (3.2). Denote $r=1+\varepsilon$ and $\log _{2}(x)=\log (\log x)$. The series

$$
\sum_{n \geq 2} \frac{\left|a_{n}\right|}{n}=\sum_{n \geq 2} \frac{1}{n(\log n)\left[\log _{2}(n)\right]^{r}}
$$

is convergent since $r>1$.

Consider the $C^{1}$ function

$$
f(x)=(\log x)\left[\log _{2}(x)\right]^{r}
$$

with

We have

$$
f^{\prime}(x)=\frac{\left(\log _{2}(x)\right)^{r}+r\left(\log _{2}(x)\right)^{r-1}}{x}
$$

$$
b_{n}=\frac{f(n+1)-f(n)}{\log (n)\left[\log _{2}(n)\right]^{r} \log (n+1)\left[\log _{2}(n+1)\right]^{r}} .
$$

For each $n$ there is a point $\theta_{n}$ between 0 and 1 such that

$$
f(n+1)-f(n)=f^{\prime}\left(n+\theta_{n}\right) .
$$

We obtain

$$
\left|b_{n}\right|=\left|\frac{\left(\log _{2}\left(n+\theta_{n}\right)\right)^{r}+r\left(\log _{2}\left(n+\theta_{n}\right)\right)^{r-1}}{\left(n+\theta_{n}\right) \log (n)\left[\log _{2}(n)\right]^{r} \log (n+1)\left[\log _{2}(n+1)\right]^{r}}\right|
$$

and thus

$$
\left|b_{n}\right| \leq \frac{1+r}{n(\log n)\left[\log _{2}(n)\right]^{r}}
$$

for sufficiently large $n$. Thus the sequence $\left(b_{n}\right)$ is absolutely summable.

Consider now the $C^{2}$ function $g(x)=1 / f(x)$, with its second derivative given by

$$
g^{\prime \prime}(x)=\frac{1}{x^{2}(\log x)^{2}\left(\log _{2} x\right)^{r}}
$$




$$
\begin{gathered}
+\frac{2}{x^{2}(\log x)^{3}\left(\log _{2} x\right)^{r}}+\frac{3 r}{x^{2}(\log x)^{3}\left(\log _{2} x\right)^{1+r}} \\
+\frac{r}{x^{2}(\log x)^{2}\left(\log _{2} x\right)^{1+r}}+\frac{r(1+r)}{x^{2}(\log x)^{3}\left(\log _{2} x\right)^{2+r}} .
\end{gathered}
$$

For each $n$, there is $\eta_{n}$ between 0 and 2 such that

$$
c_{n}=g(n)-2 g(n+1)+g(n+2)=g^{\prime \prime}\left(n+\eta_{n}\right) .
$$

Using this representation of $c_{n}$ it can be proved that $\left(n c_{n}\right)$ is absolutely summable.

\section{A sufficient CONDition for similarity of $R(X)$ to $R(0)$}

The idea of the proof of the following theorem goes back to $[\mathrm{FW}]$ and $[\mathrm{W}]$.

Theorem 4.1. Let $T_{2} \in B\left(H_{2}\right)$ be an isometry $\left(T_{2}^{*} T_{2}=I_{H_{2}}\right)$ and let $T_{1} \in B\left(H_{1}\right)$ and $X: H_{1} \rightarrow H_{2}$ be bounded operators. Consider

$$
R\left(T_{2}^{*}, T_{1} ; X\right)=\left[\begin{array}{cc}
T_{2}^{*} & X \\
0 & T_{1}
\end{array}\right]
$$

If

$$
\sup _{n \geq 1}\left\|\sum_{j=0}^{n-1} T_{2}^{j+1} X T_{1}^{j}\right\|<+\infty
$$

then $R\left(T_{2}^{*}, T_{1} ; X\right)$ is similar to $T_{2}^{*} \oplus T_{1}=R\left(T_{2}^{*}, T_{1} ; 0\right)$.

Proof. Let $\mathcal{L}$ be a Banach limit $[\mathrm{C}]$, that is a bounded linear functional on $\ell_{\infty}(\mathbb{C})$ such that $\mathbf{1}=\mathcal{L}(\mathbf{1})=\|\mathcal{L}\|$ and $\mathcal{L}\left(\left(x_{n+1}\right)_{n \geq 0}\right)=\mathcal{L}\left(\left(x_{n}\right)_{n \geq 0}\right)$ for every $\left(x_{n}\right)_{n \geq 0} \in \ell_{\infty}(\mathbb{C})$. Here $\mathbf{1}=(1,1, \ldots)$.

Consider the linear operator $Z: H_{1} \rightarrow H_{2}$ given by

$$
\left\langle Z h_{1}, h_{2}\right\rangle_{H_{2}}=\mathcal{L}\left(\left\langle\sum_{j=0}^{n-1} T_{2}^{j+1} X T_{1}^{j} h_{1}, h_{2}\right\rangle\right) .
$$

Then (4.1) shows that $Z$ is well-defined and bounded. 
We have

$$
\begin{aligned}
\left\langle\left(T_{2}^{*} Z-Z T_{1}\right) h_{1}, h_{2}\right\rangle= & \left\langle Z h_{1}, T_{2} h_{2}\right\rangle-\left\langle Z T_{1} h_{1}, h_{2}\right\rangle \\
= & \mathcal{L}\left(\left\langle\sum_{j=0}^{n-1} T_{2}^{j+1} X T_{1}^{j} h_{1}, T_{2} h_{2}\right\rangle\right. \\
& \left.-\left\langle\sum_{j=0}^{n-1} T_{2}^{j+1} X T_{1}^{j+1} h_{1}, h_{2}\right\rangle\right) \\
= & \mathcal{L}\left(\left\langle\sum_{j=0}^{n-1} T_{2}^{j} X T_{1}^{j} h_{1}, h_{2}\right\rangle\right. \\
& \left.-\left\langle\sum_{j=0}^{n-1} T_{2}^{j+1} X T_{1}^{j+1} h_{1}, h_{2}\right\rangle\right) \\
= & \left\langle X h_{1}, h_{2}\right\rangle-\mathcal{L}\left(\left\langle T_{2}^{n} X T_{1}^{n} h_{1}, h_{2}\right\rangle\right) .
\end{aligned}
$$

On the other hand,

$$
\begin{aligned}
\mathcal{L}\left(\left\langle T_{2}^{n} X T_{1}^{n} h_{1}, h_{2}\right\rangle\right)= & \mathcal{L}\left(\left\langle T_{2}^{n} X T_{1}^{n} h_{1}, T_{2}^{*} T_{2} h_{2}\right\rangle\right) \\
= & \mathcal{L}\left(\left\langle T_{2}^{n+1} X T_{1}^{n} h_{1}, T_{2} h_{2}\right\rangle\right) \\
= & \mathcal{L}\left(\left\langle\sum_{j=0}^{n} T_{2}^{j+1} X T_{1}^{j} h_{1}, T_{2} h_{2}\right\rangle\right) \\
& -\mathcal{L}\left(\left\langle\sum_{j=0}^{n-1} T_{2}^{j+1} X T_{1}^{j} h_{1}, T_{2} h_{2}\right\rangle\right) \\
= & 0 .
\end{aligned}
$$

Therefore $T_{2}^{*} Z-Z T_{1}=X$ and thus

$$
\left[\begin{array}{cc}
I & -Z \\
0 & I
\end{array}\right]\left[\begin{array}{cc}
T_{2}^{*} & 0 \\
0 & T_{1}
\end{array}\right]\left[\begin{array}{ll}
I & Z \\
0 & I
\end{array}\right]=\left[\begin{array}{cc}
T_{2}^{*} & X \\
0 & T_{1}
\end{array}\right] .
$$

We obtain that $R\left(T_{2}^{*}, T_{1} ; X\right)$ is similar to $T_{2}^{*} \oplus T_{1}=R\left(T_{2}^{*}, T_{1} ; 0\right)$.

It follows from the above Theorem that if (4.1) holds and $T_{1}$ is similar to a contraction, then $R\left(T_{2}^{*}, T_{1} ; X\right)$ is similar to a contraction. We refer to $[\mathrm{FW}, \mathrm{C}+3, \mathrm{Cl}, \mathrm{Pa} 3, \mathrm{Ca}]$ for related results. 
Corollary 4.2. Let $T_{2} \in B\left(H_{2}\right)$ be an isometry and let $T_{1} \in B\left(H_{1}\right)$ and $X: H_{1} \rightarrow H_{2}$ be bounded operators such that the spectral radius of $T_{1}$ satisfies $r\left(T_{1}\right)<1$. Then

$$
R\left(T_{2}^{*}, T_{1} ; X\right)=\left[\begin{array}{cc}
T_{2}^{*} & X \\
0 & T_{1}
\end{array}\right]
$$

is similar to a contraction.

Proof. We have

$$
\begin{gathered}
\left\|\sum_{j=0}^{n-1} T_{2}^{j+1} X T_{1}^{j}\right\| \leq \sum_{j=0}^{n-1}\left\|T_{2}^{j+1}\right\|\|X\|\left\|T_{1}^{j}\right\| \\
\leq\|X\| \sum_{j=0}^{+\infty}\left\|T_{1}^{j}\right\|
\end{gathered}
$$

for each $n$. The last sum converges since $r\left(T_{1}\right)<1$. By Theorem 4.1, $R\left(T_{2}^{*}, T_{1} ; X\right)$ is similar to $T_{2}^{*} \oplus T_{1}$. The operator $T_{2}^{*}$ is a contraction, while $T_{1}$ is similar to a contraction by Rota's [Ro] theorem. Thus $T_{2}^{*} \oplus T_{1}$ is similar to a contraction.

The following simple result characterizes when $R\left(T_{2}^{*}, T_{1} ; X\right)$ is powerbounded in terms of a condition related to (4.1) and the power-boundedness of $T_{1}$.

Proposition 4.3. Let $T_{2} \in B\left(H_{2}\right)$ be an isometry $\left(T_{2}^{*} T_{2}=I_{H_{2}}\right)$ and let $T_{1} \in B\left(H_{1}\right)$ and $X: H_{1} \rightarrow H_{2}$ be bounded operators. Then

$$
R\left(T_{2}^{*}, T_{1} ; X\right)=\left[\begin{array}{cc}
T_{2}^{*} & X \\
0 & T_{1}
\end{array}\right] .
$$

is power-bounded if and only if

$$
\sup _{n \geq 1}\left\|T_{2}^{* n} \sum_{j=0}^{n-1} T_{2}^{j+1} X T_{1}^{j}\right\|<+\infty .
$$

and $T_{1}$ is power-bounded.

Proof. Set $R=R\left(T_{2}^{*}, T_{1} ; X\right)$. We have

$$
R^{n}=\left[\begin{array}{cc}
T_{2}^{* n} & \sum_{j=0}^{n-1} T_{2}^{*(n-j-1)} X T_{1}^{j} \\
0 & T_{1}^{n}
\end{array}\right] .
$$


Since $T_{2}$ is an isometry, we have

$$
\sum_{j=0}^{n-1} T_{2}^{*(n-j-1)} X T_{1}^{j}=T_{2}^{* n} \sum_{j=0}^{n-1} T_{2}^{j+1} X T_{1}^{j}
$$

which gives the desired equivalence.

\section{Proofs of the main Results}

Proof of Theorem 2.1. By Theorem 4.1, $R\left(S^{*(\infty)}, S^{(\infty)} ; X\right)$ is similar to a contraction whenever

$$
\sup _{n \geq 1}\left\|\sum_{k=0}^{n-1} S^{(\infty)(k+1)} X S^{(\infty) k}\right\|<+\infty .
$$

The matrix of the operator $S^{(\infty) k}$ is given by

$$
\left[\begin{array}{cccc}
0 & 0 & 0 & \cdots \\
\vdots & \vdots & \vdots & \cdots \\
0 & 0 & 0 & \cdots \\
1 & 0 & 0 & \cdots \\
0 & 1 & 0 & \cdots \\
0 & 0 & 1 & \cdots \\
\vdots & \vdots & \vdots & \ddots
\end{array}\right]
$$

where the first non-zero entry occurs at position $(k, 0)$. The matrix of the operator $S^{(\infty)(k+1)} X S^{(\infty) k}$ is

$$
\left[\begin{array}{cccc}
0 & 0 & 0 & \cdots \\
\vdots & \vdots & \vdots & \cdots \\
0 & 0 & 0 & \cdots \\
X_{0 k} & X_{0, k+1} & X_{0, k+2} & \cdots \\
X_{1, k} & X_{1, k+1} & X_{1, k+2} & \cdots \\
\vdots & \vdots & \vdots & \ddots
\end{array}\right]
$$

where the first non-zero entry occurs at position $(k+1,0)$. This gives that the entries of the matrix $\tilde{A}^{(n)}(X)$ of the operator

$$
\sum_{k=0}^{n-1} S^{(\infty)(k+1)} X S^{(\infty) k}
$$


are 0 on the first line and

$$
\tilde{A}^{(n)}(X)_{i j}=X_{i-1, j}+X_{i-2, j+1}+\ldots X_{i-1-\min (i-1, n-1), j+\min (i-1, n-1)}
$$

for $i \geq 1$. Then the operator represented by $\tilde{A}^{n}(X)$ is bounded whenever the operator represented by $A^{(n)}(X)$ is.

Proof of Theorem 2.2. By [FW], the Foguel-Hankel operator

$$
R(\Gamma)=R\left(S^{*(\infty)}, S^{(\infty)} ; \Gamma\right)
$$

is similar to a contraction if and only if there is a bounded solution $Y$ of the equation

$$
S^{*(\infty)} Y-Y S^{(\infty)}=\Gamma,
$$

if and only if $R(\Gamma)$ is similar to $S^{*(\infty)} \oplus S^{(\infty)}$. One completes the proof by observing that each of the three operators, $-\Gamma D, D^{*} \Gamma$ and $(-\Gamma D+$ $\left.D^{*} \Gamma\right) / 2$ is a formal solution to this commutator equation.

In the case of a scalar symbol $\Gamma_{f}$, one can say considerably more. If a matrix $Y$ is any formal solution(bounded or unbounded) of (5.1), then $-Y^{t}$ is another solution of the same(possibly infinite) norm. Here $Y^{t}$ is the transpose of $Y$. Indeed, $\left(\Gamma_{f}\right)^{t}=\Gamma_{f}$ and $S^{*}=S^{t}$. Hence $\left[Y-Y^{t}\right] / 2$ is a solution of no greater norm. Now if $Y$ is a solution of (5.1), then any other solution differs from $Y$ by a matrix $G$ satisfying

$$
S^{*} G=G S
$$

that is by a formal Hankel matrix $G$. But

$$
(Y+G)-(Y+G)^{t}=Y-Y^{t}
$$

and so if $Y$ is any solution of (5.1), then $\left[Y-Y^{t}\right] / 2$ is the solution of minimum norm.

Now

$$
Y_{0}=-\Gamma_{f} D=\left[-j a_{i+j-1}\right]_{i, j \geq 0}
$$

is a formal solution of (5.1) and thus

$$
\left(\left[\frac{(j-i) a_{i+j-1}}{2}\right]_{i, j \geq 0}\right)^{t}=\frac{\left(\Gamma_{f} D-D^{*} \Gamma_{f}\right)^{t}}{2}=\frac{\left[Y_{0}-Y_{0}^{t}\right]}{2}
$$

is the solution of minimum norm. 
Thus, we see immediately as an application of $[\mathrm{FW}]$ that $R\left(\Gamma_{f}\right)$ is similar to a contraction if and only if $\Gamma_{f} D-D^{*} \Gamma_{f}$ is bounded.

Unfortunately, the above arguement fails in the operator-valued Hankel setting. The difficulty is that the transpose of a bounded operator matrix need not be bounded. This is essentially because the transpose map is not completely bounded. For this reason it is not known what the minimum norm solution of (5.1) is in the operator case. This makes it difficult to obtain necessary and sufficient conditions for the existence of bounded solutions.

Proof of Theorem 2.3. By Theorem 2.2 it is sufficient to show that

$$
\left[(j-i) \alpha_{i+j-1} C_{i+j-1}\right]_{i, j \geq 1}
$$

is bounded. Indeed, this matrix is obtained from the matrix

$$
\left[(j-i) \alpha_{i+j-1} C_{i+j-1}\right]_{i, j \geq 0}
$$

of Theorem 2.2 by adding a bounded row and column.

Let $\varepsilon>0$. The matrix $\left[(j-i) \alpha_{i+j-1} C_{i+j-1}\right]_{i, j \geq 1}$ can be viewed as the Schur product of $F_{\varepsilon / 2}$ and

$$
\left[(i+j+1)(\log (i+j+1))(\log (\log (i+j+1)))^{(\varepsilon+2) / 2} \alpha_{i+j-1} C_{i+j-1}\right]_{i, j \geq 1} .
$$

The last matrix represents a bounded Hankel operator. Indeed, we have $[\mathrm{DP}]$

$$
\begin{gathered}
\left\|\left[(i+j+1)(\log (i+j+1))(\log (\log (i+j+1)))^{(\varepsilon+2) / 2} \alpha_{i+j-1} C_{i+j-1}\right]\right\| \\
=\left\|\left[(i+j+1)(\log (i+j+1))(\log (\log (i+j+1)))^{(\varepsilon+2) / 2} \alpha_{i+j-1} e_{i+j-1}\right]\right\| \\
=\sum_{k \geq 1}(k+2)^{2}(\log (k+2))^{2}\left[\log (\log (k+2)]^{2+\varepsilon}\left|\alpha_{k}\right|^{2}\right.
\end{gathered}
$$

which is convergent.

Since $F_{\varepsilon / 2}$ is a Schur multiplier by Corollary 3.2, their Schur product gives [DP, Theorem 4.1] a bounded operator. 


\section{REFERENCES}

[AP] A.B. Aleksandrov and V.V. Peller : Hankel operators and similarity to a contraction, Internat. Math. Res. Notices 1996, no. 6, 263-275.

[Ba] C. Badea : Operators near completely polynomially dominated ones and similarity problems, to appear in J. Operator Th.

[Be] G. Bennett : Schur multipliers, Duke Math. J. 44(1977), 603-639.

[Bl] G. Blower : Multipliers of Hardy spaces, quadratic integrals and FoiasWilliams-Peller operators, Studia Math. 131(1998), 179-188.

[B] J. Bourgain : On the similarity problem for polynomially bounded operators, Israel J. Math. 54(1986), 227-241.

[Bo] M. Bożejko : Littlewood functions, Hankel multipliers and power bounded operators, Colloq. Math. 51(1987), 35-42.

[C+3] J.F. Carlson, D.N. Clark, C. Foias and J.P. Williams : Projective Hilbert A(D)-modules, New York J. Math. 1(1994), 26-38.

[Ca] G. Cassier : Generalized Toeplitz operators and similarity problems, Preprint.

[Cl] D.N. Clark : Projectivity and lifting of Hilbert module maps, Ann. Polon. Math. 66(1997), 43-48.

[C] J.B. Conway : A Course in Functional Analysis, Second edition. Graduate Texts in Mathematics, 96. Springer-Verlag, New York, 1990.

[D] K.R. Davidson : Polynomially bounded operators, a survey, in : Operator algebras and applications (Samos, 1996), 145-162, Kluwer Acad. Publ., Dordrecht, 1997.

[DP] K.R. Davidson ; V. I. Paulsen : Polynomially bounded operators, J. Reine Angew. Math. 487(1997), 153-170.

[Da] A.M. Davie : Power-bounded elements in a $Q$-algebra, Bull. London Math. Soc. 6 (1974), 61-65.

[Fe] S. Ferguson : Polynomially bounded operators and Ext groups, Proc. Amer. Math. Soc. 124(1996), 2779-2785.

[F] S. Foguel : A counterexample to a problem of Sz.-Nagy, Proc. Amer. Math. Soc. 15(1964), 788-790.

[FW] C. Foias and J. Williams : On a class of polynomially bounded operators, Preprint 1980.

[JP] S. Janson ; J. Peetre : Paracommutators-boundedness and Schatten-von Neumann properties, Trans. Amer. Math. Soc. 305 (1988), 467-504.

[L] A. Lebow : A power bounded operator which is not polynomially bounded, Michigan Math. J.. 15 (1968), 397-399.

[Pa1] V. I. Paulsen : Every completely polynomially bounded operator is similar to a contraction, J. Funct. Anal. 55(1984), 1-17.

[Pa2] V. I. Paulsen : Completely Bounded Maps and Dilations, Longman, 1986. 
[Pa3] V. I. Paulsen : Relative Yoneda cohomology for operator spaces, J. Funct. Anal. 157 (1998), 358-393.

[Pe1] V.V. Peller : Estimates of functions of power bounded operators in Hilbert spaces, J. Operator Th. 7 (1982), 341-372.

[Pe2] V.V. Peller : Estimates of functions of Hilbert space operators, similarity to a contraction and related function algebras, Lect. Notes Math. 1573, 298-302, Springer-Verlag, Berlin, 1984.

[P] S. Petrović : Some remarks on the operator of Foias and Williams, Proc. Amer. Math. Soc. 124(1996), 2807-2811.

[Pi] G. Pisier : A polynomially bounded operator on Hilbert space which is not similar to a contraction, J. Amer. Math. Soc. 10(1997), 351-369.

[Ro] G.C. Rota : On models for linear operators, Comm. Pure Appl. Math. 13(1960), 469-472.

[vN] J. von Neumann : Eine Spektraltheorie für allgemeine Operatoren eines unitären Raumes, Math. Nachr. 4(1951), 258-281.

[W] J.P. Williams : On the range of a derivation. II, Proc. Roy. Irish Acad. Sect. A 74 (1974), 299-310.

Mathématiques, UMR 8524 au CNRS, Université de Lille I, F-59655

Villeneuve D'AscQ, France

E-mail address: catalin.badea@agat.univ-lille1.fr

Department of Mathematics, University of Houston, Houston, TX 77204-3476

E-mail address: vern@math.uh.edu 